\title{
The Lateral Tarsal Strip Procedure: Reflections by a Specialist With 20 Years of Experience in a Tertiary Medical Center (Cross-sectional Study)
}

Jonghyun Kim

Korea University College of Medicine

Joohyun Kim

Korea University College of Medicine

Sehyun Baek ( $\sim$ shbaek6534@korea.ac.kr)

Korea University College of Medicine

\section{Research Article}

Keywords: Horizontal laxity, Involutional ectropion, Involutional entropion, Lateral tarsal strip

Posted Date: January 3rd, 2022

DOI: https://doi.org/10.21203/rs.3.rs-1194419/v1

License: (c) (i) This work is licensed under a Creative Commons Attribution 4.0 International License.

Read Full License 


\section{Abstract}

Introduction: Lateral tarsal strip (LTS) is a simple surgical technique that can correct eyelid malposition. Clinical indications vary from involutional entropion and ectropion, lower lid laxity, and lower lid retraction to punctal and paralytic ectropion. LTS mainly treats eyelid malposition by correcting horizontal laxity. Herein, we report the clinical indications for and effects of LTS.

Methods: We retrospectively reviewed medical records of patients who underwent LTS by a single oculoplastic surgeon with eight years of experience performing the procedure at Korea University Guro Hospital.

Results: We included 247 patients with 325 treated eyelids in the study. Involutional entropion was the most common indication, accounting for 69 patients (27.94\%) and 88 eyelids (27.08\%). The second most common indication was lower lid laxity ( $\mathrm{n}=44$ patients $(17.81 \%)$ and 68 eyelids $(20.92 \%)$ ). Simultaneous surgery in addition to LTS was performed in 167 patients (67.6\%) and 219 eyelids (67.4\%); the most common was endoscopic dacryocystorhinostomy (DCR), which was performed in 50 patients $(29.94 \%)$ and 80 eyelids $(36.53 \%)$.

Conclusion: LTS can be performed alone or simultaneously with oculoplastic procedures for various indications. Overcorrection with fixation above the canthal angle is useful to reduce recurrence. This study aims to explain the clinical importance of the LTS procedure.

\section{Introduction}

Lateral tarsal strip (LTS) is a surgical approach performed worldwide to correct eyelid malposition. In particular, LTS corrects lateral canthal tendon laxity.(1) The main pathologic factor of lower eyelid malposition is horizontal laxity or elongation. Eyelid tightening with lateral canthal tendon stabilization by LTS can resolve these problems.(2)

Advantages like procedure simplicity and short surgery time allow LTS to be performed with other oculoplastic surgeries. For example, patients with lower eyelid laxity and nasolacrimal duct obstruction can experience improved lacrimal pump mechanism with LTS and endoscopic dacryocystorhinostomy. Also, LTS can resolve malposition for involutional ectropion patients without medial canthus laxity.(3-5) Furthermore, LTS can treat lower eyelid horizontal laxity and conjunctival fornix contracture associated with ocular prosthesis in anopia patients.(6)

There are several modified LTS approaches that have been compared with the simple LTS procedure. $(2,5$, 7-11) However, patients with lower eyelid malposition due to horizontal laxity are generally of older age, and a relatively short surgery time with accurate eyelid correction is important for these patients. Therefore, in this study, we report the clinical indications and outcomes from LTS by retrospectively investigating patient medical records. 


\section{Methods}

\section{Ethics statement}

This study was approved by the Institutional Review Board of Korea University in Seoul, Korea (K20211260-001). Patient informed consent was obtained from all patients and/or their legal guardians. Data collection and analyses were conducted in accordance with the tenets of the Declaration of Helsinki from the Word Medical Association.

\section{Patients}

We reviewed the medical records of consecutive patients who underwent LTS between March 2, 2014, and February 28, 2021, at Korea University Guro Hospital. LTS was performed by one oculoplastic surgery expert who had been conducting oculoplastic surgeries for over 20 years (S.B.).

Patients were included if they underwent LTS on their unilateral or bilateral lower eyelids and were diagnosed by an oculoplastic specialist as having oculoplastic disease. Patients were excluded if they underwent LTS secondary to canthotomy or cantholysis with reconstruction for facial bone fracture (e.g., blow-out fracture). Totals of 247 patients and 325 eyelids were included in this study.

Patients' medical and surgical records were reviewed, and demographic and clinical data were extracted: sex, age, and follow-up duration (months) were included. Patient population data were reported, such as number of patients and eyelids and percentage of unilateral or bilateral eyelids. Clinical indications and simultaneous surgical procedures were determined from diagnoses recorded in outpatient and surgical records. Any inconsistencies between the diagnosis from outpatient and surgical records were resolved by oculoplastic surgeons who reviewed the cases and made a final joint decision to reduce selection bias.

\section{Surgical technique}

All surgical procedures were performed by the same oculoplastic surgeon. Lidocaine $2 \%$ with epinephrine $1: 100,000$ was infiltrated into the lateral third of the lower eyelid. We also infiltrated into the lateral canthus including the deep orbicularis muscle and connective tissues anterior to the lateral orbital rim and into the periosteum of the lateral orbital rim. To avoid involvement of the posterior orbit and globe, palpation of the orbital rim was necessary. We recommend gently applying moderate pressure to the area of anesthetic infiltration to prevent hematoma or ecchymosis.

Lateral canthotomy and inferior cantholysis were initially performed (Fig. 1A). The lower eyelid was separated from the lateral orbital rim by cutting the lower lateral canthal ligament. A longer incision was necessary for improved access to the orbital rim. We then grasped the most lateral aspect of the lower eyelid with Adson's forceps and applied traction to the orbital rim to allow free movement of the lower eyelid (Fig. 1B). The incision can be made deep into the orbicularis muscle to the orbital rim using curved Steven's scissors or Wescott scissors. 
A lateral tarsal strip is made from the lateral lower eyelid by stripping the mucocutaneous junction tissue, removing the conjunctival epithelium posteriorly, and excising the anterior lamella (Fig. 1C). The tarsal strip is firmly grasped with an Adson's forceps and pulled onto the lateral orbital rim. This procedure will provide the surgeon the approximate length of tarsal strip needed. Generally, $2 \mathrm{~mm}$ above the lateral canthal angle is the ideal point for the LTS suture site. The aim is to slightly overcorrect the height of the eyelid to maintain the posterior tension of the lower eyelid.

The lateral tarsal strip is attached to the internal lateral orbital rim with a double-armed 4-0 polyglactin suture (Fig. 1D). The needle is passed through the full thickness of the superolateral aspect of tarsal strip and sutured to the periosteum above the original location of the lower eyelid. A mattress suture is then made after passing the thread through the posterior aspect of the upper eyelid. A deeply buried 7-0 polyglactin suture is used to close the lateral canthal orbicularis muscle. Skin closure follows with interrupted 7-0 black silk sutures.

Antibiotic ofloxacin ointment was applied on the lateral suture site and lower conjunctival fornix, and hydrophilic polyurethane foam was applied to the skin suture site. Patients were recommended to apply levofloxacin eye drops four times a day for one week. Also, patients were asked not to pull their lower eyelid when applying eye drops to prevent wound dehiscence. Applying a cold pack for 3 days and then a hot pack for 2 days consecutively was important to reduce eyelid swelling and improve wound healing. The first postoperative review in the outpatient clinic was one week after surgery. Patients were reviewed again at 1,2, and 6 months postoperatively. All patients had preoperative and postoperative clinical photos taken at every outpatient visit.

\section{Results}

Totals of 247 patients and 325 eyelids were included in this study. Patients' general characteristics are presented in Table 1. Briefly, 124 (50.2\%) were male and 123 (49.8\%) were female. Unilateral eyelid LTS was performed in 169 (68.4\%) patients and bilateral eyelid LTS was performed in 78 (31.6\%) patients. Mean patient age was $61.8 \pm 16.7$ years and the follow-up duration was $12.4 \pm 17.0$ months.

Table 1

Demographics of patients undergoing lateral tarsal strip procedure

\begin{tabular}{|ll|}
\hline Patients (Eyelids) & $247(325)$ \\
\hline Male (n, \%) / Female (n, \%) & $124(50.2 \%) / 123(49.8 \%)$ \\
\hline Unilateral (n, \%) / Bilateral (n, \%) & $169(68.4 \%) / 78(31.6 \%)$ \\
\hline Age (years) & $61.8 \pm 16.7$ \\
\hline Follow-up duration (months) & $12.4 \pm 17.0$ \\
\hline Values are presented as mean \pm SD unless otherwise indicated. \\
\hline
\end{tabular}


The clinical indications of the 247 patients and 325 eyelids who underwent LTS are presented in Table 2. Involutional entropion was the most frequent indication, with 69 patients $(27.94 \%)$ and 88 eyelids (27.08\%), followed by lower lid laxity (44 patients (17.81\%) and 68 eyelids (20.92\%)), lower lid retraction (44 patients $(17.81 \%)$ and 60 eyelids $(18.46 \%)$ ), involutional ectropion (28 patients $(11.34 \%)$ and 35 eyelids $(10.77 \%)$ ), cicatricial ectropion (12 patients (4.86\%) and 16 eyelids $(4.92 \%)$ ), and punctal ectropion (11 patients $(4.45 \%)$ and 16 eyelids( $4.92 \%))$.

Table 2

Clinical indications of the lateral tarsal strip procedure

\begin{tabular}{|lll|}
\hline Diagnosis & Patients (n, \%) & Eyelids (n, \%) \\
\hline Involutional entropion & $69(27.94 \%)$ & $88(27.08 \%)$ \\
\hline Lower lid laxity & $44(17.81 \%)$ & $68(20.92 \%)$ \\
\hline Lower lid retraction & $44(17.81 \%)$ & $60(18.46 \%)$ \\
\hline Involutional ectropion & $28(11.34 \%)$ & $35(10.77 \%)$ \\
\hline Cicatricial ectropion & $12(4.86 \%)$ & $16(4.92 \%)$ \\
\hline Punctal ectropion & $11(4.45 \%)$ & $16(4.92 \%)$ \\
\hline Paralytic ectropion & $8(3.24 \%)$ & $9(2.77 \%)$ \\
\hline Lateral canthal deformity & $8(3.24 \%)$ & $10(3.08 \%)$ \\
\hline Socket contracture & $5(2.02 \%)$ & $5(1.54 \%)$ \\
\hline Cicatricial entropion & $1(0.40 \%)$ & $17(5.23 \%)$ \\
\hline Others * & $17(6.88 \%)$ & 325 \\
\hline Total & 247 & \\
\hline * Others include skin defects of the lower eyelid secondary to tumor excision and traumatic lower \\
eyelid laceration.
\end{tabular}

Among the LTS patients, 167 (67.6\%) and 219 eyelids (67.4\%) underwent a second simultaneous surgery. Endoscopic dacryocystorhinostomy (DCR) was the most frequent simultaneous surgery and was performed in 50 patients $(29.94 \%$ ) and 80 eyelids (36.53\%), followed by skin muscle excision (29 patients (17.37\%) and 34 eyelids (15.53\%)), inferior retractor tightening (29 patients $(17.37 \%)$ and 34 eyelids $(15.53 \%)$ ), mid-lamellar lengthening (24 patients (14.37\%) and 30 eyelids (13.70\%)), and endoscopic conjunctivodacryocystorhinostomy (CDCR) (11 patients (6.59\%) and 13 eyelids (5.94\%)) (Table 3 ). 
Table 3

Surgical procedures performed simultaneously with lateral tarsal strip procedure

\begin{tabular}{|lll|}
\hline & Patients $(\mathbf{n}, \%)$ & Eyelids (n, \%) \\
\hline Endoscopic DCR & $50(29.94 \%)$ & $80(36.53 \%)$ \\
\hline Skin muscle excision & $29(17.37 \%)$ & $34(15.53 \%)$ \\
\hline Inferior retractor tightening & $29(17.37 \%)$ & $34(15.53 \%)$ \\
\hline Mid-lamellar lengthening & $24(14.37 \%)$ & $30(13.70 \%)$ \\
\hline Endoscopic CDCR & $11(6.59 \%)$ & $13(5.94 \%)$ \\
\hline Socket reconstruction & $3(1.80 \%)$ & $3(1.37 \%)$ \\
\hline Primary repair of canalicular laceration & $2(1.20 \%)$ & $2(0.91 \%)$ \\
\hline Medial spindle procedure & $2(1.20 \%)$ & $3(1.37 \%)$ \\
\hline Lower blepharoplasty & $2(1.20 \%)$ & $4(1.83 \%)$ \\
\hline Others* & $14(8.38 \%)$ & $14(6.39 \%)$ \\
\hline Total & 167 & 219 \\
\hline DCR = dacryocystorhinostomy, CDCR = conjunctivodacryocystorhinostomy & \\
\hline $\begin{array}{l}* \text { OOthers include Tenzel semicircular rotation flap, skin graft secondary to tumor excision, lateral } \\
\text { tarsorhaphy, partial tarsorrhaphy, root-z epicanthoplasty, scar release, transconjunctival fat removal, } \\
\text { pentagonal wedge excision, Hotz operation. }\end{array}$ & \\
\hline
\end{tabular}

\section{Discussion}

This study shows that lateral tarsal strip (LTS) procedure can be applied solely or simultaneously with other oculoplastic surgeries for indications like involutional entropion, lower lid laxity, lower lid retraction, and nasolacrimal duct obstruction.

Entropion and ectropion are ophthalmological terms for defects of eyelid positioning.(12) Entropion is unusual coiling of the lower eyelid margin, and ectropion is malposition of the eyelid in which the lower eyelid falls away from its normal position to the orbit, exposing the palpebral and conjunctiva. $(2,12)$ Involutional entropion and ectropion are the most common types of eyelid malposition seen in ophthalmology practices. In this study population, involutional lid factors accounted for about $40 \%$ of clinical indications for LTS procedure. Paralytic and cicatricial etiologies were rare (Table 2).

Involutional entropion is generally caused by horizontal laxity and a combination of lower eyelid retractor dehiscence and orbicularis muscle override.(11) The standard surgical correction for these conditions is the LTS procedure. LTS can address both tarsal lengthening and lateral canthal tendon laxity. By firmly 
attaching the tarsal strip to the internal lateral orbital rim, this procedure offers better fixation and helps prevent outward or inward rotation of the inferior tarsal plate and orbicularis overriding.(2)

The amount of lateral tarsal strip tightening required depends on the height of the fixation point at the internal orbital rim; we recommend $2 \mathrm{~mm}$ above the lateral canthal angle (Fig. 2). A patient in our study who was concerned about unnatural correction of lower lid laxity underwent LTS lower than the usual fixation height. Three months after the surgery, the patient revisited our clinic because of recurrence (Fig. 3). Undercorrection with lower LTS fixation height is the main cause of recurrence. Thus, we performed revision of the lateral tarsal strip above the first fixation point, and there was no further recurrence.

Chang et al. proposed a useful augmented lateral tarsal strip tarsorrhaphy for paralytic ectropion.(7) During augmented LTS, the periosteum on the lateral orbital rim is exposed to higher than standard LTS. The longer tarsal strip is attached $5 \mathrm{~mm}-8 \mathrm{~mm}$ above the horizontal intercanthal line. The augmented tarsal strip produces a greater elevating effect through a longer strip attached to the higher internal orbital rim for paralytic ectropion.(7) We also suggest overcorrection of the lateral tarsal strip for lagophthalmos and lower lid retraction to reduce recurrence (Fig. 4). Overcorrection effectively reduces the vertical palpebral aperture when LTS alone is insufficient. Patients should be warned that the lower eyelid can appear very tight and over elevated. These initial unnatural appearances will decrease within several weeks to a stable position, and patients usually achieve good symmetry about six months after surgery.

Some authors suggest other procedures like the medial spindle procedure to correct ectropion along with the LTS procedure. $(13,14)$ However, we argue that LTS alone can sufficiently achieve both functional and anatomical success. Kam et al. compared symptomatic and anatomical improvement between LTS alone and LTS with medial spindle procedure. Functional and anatomical results did not differ significantly between the two operations.(5) Surgeons might overuse the medial spindle, and LTS alone is sufficient for correcting the vast majority of involutional ectropion cases. Advantages of performing LTS alone are speed, no danger of lid margin notching, minimal removal of lid tissue, and simultaneous correction of canthal malposition and lid shortening.(5) A short surgery time is crucial for a better prognosis in elderly patients because they cannot endure long periods in an uncomfortable position. Also, in consideration of a delayed wound repair process, minimally invasive procedures are needed for elderly patients.

To correct lower lid retractor disinsertion and orbicularis override, which also induce tarsal instability, skin muscle excision on the lower lid is important for correcting involutional ectropion (Fig. 5). Compared with a transconjunctival approach, the external approach using skin muscle excision can reduce entropion recurrence and create a cicatricial force during skin healing.(9) In this study, 29 patients (17.37\%) with 34 eyelids (15.53\%) who underwent skin muscle excision were the second most common group of patients receiving simultaneous surgeries (Table 3 ).

Functional epiphora is indicated as epiphora with no anatomical outflow blockage. In epiphora patients without lacrimal punctum obstruction and nasolacrimal duct stenosis, lacrimal pump dysfunction is the cause. $(15,16)$ Dysfunction of the lacrimal pump is generated by lower lid laxity, lower lid retraction, and 
orbicularis oculi muscle paralysis. LTS can strengthen the horizontal power of the lower eyelid and improve lacrimal pump function.(8) Also, LTS can improve functional success because it can restore the lower lid from the orbit, presenting the tear reservoir to the upper canaliculus during blinking and allowing proper drainage to the nasolacrimal duct.(5) In this study, 44 patients $(17.81 \%)$ with lower lid laxity (Fig. 6) and 44 patients (17.81\%) with lower lid retraction (Fig. 7) underwent LTS, and 50 patients (29.94\%) underwent simultaneous LTS and endoscopic dacryocystorhinostomy (Tables 2 \& 3).

Lateral tarsal strip is a relatively simple procedure, and complications are rare. The most common complaint from patients is mild tenderness and serous discharge at the lateral canthal site. These complaints mostly resolve within 1-2 weeks post-surgery. Other rare complications are lateral webbing (Fig. 8) and wound dehiscence. Patients undergoing LTS should be cautioned not to rub and stretch the tight lower eyelid when applying eye drops and ointments. Also, if the tarsal strip is placed too anteriorly, a gap between the eyelid and orbit might occur. To prevent this, modified Frost suture, which is a 6-0 silk suture passed through the eyelid margin and attached to the forehead, is useful.(17)

Limitations of this study include its retrospective and noncomparative nature, as well as a short follow-up period, which was a consequence of the population's inability to comply with multiple postoperative visits.

In summary, lateral tarsal strip is a simple technique that allows tightening of the lower eyelid to correct horizontal laxity. LTS can be applied alone or simultaneously with oculoplastic procedures for various indications. To reduce recurrence rates, overcorrection with fixation of the lateral tarsal strip above the canthal angle is important.

\section{Abbreviations}

LTS

lateral tarsal strip

CDCR

endoscopic conjunctivodacryocystorhinostomy

\section{Declarations}

Ethics approval and consent to participate : This study was approved by the Institutional Review Board of Korea University in Seoul, Korea (K2021-1260-001). Patient informed consent was obtained from all patients and/or their legal guardians. Data collection and analyses were conducted in accordance with the tenets of the Declaration of Helsinki from the Word Medical Association.

Consent for publication : Patient informed consent was received by the patient and/or their legal guardians. 
Availability of data and materials : Data sharing is not applicable to this article as no datasets were generated or analyzed during the current study

Competing interests : The authors declare that they have no competing interests

Funding: The authors have no financial support or financial conflicts of interest to declare.

Authors' contributions

Conception and design of the study (J.H., J.H., S.B.);

Conduct of the study (J.H., J.H., S.B.);

Collection and management of the data (J.H., J.H., S.B.);

Data analysis (J.H., J.H., S.B.);

Data interpretation (J.H., J.H., S.B.);

Preparation, review, and approval of the manuscript (J.H., J.H., S.B.)

Acknowledgements : Not applicable

\section{References}

1. Anderson RL, Gordy DD. The tarsal strip procedure. Archives of ophthalmology (Chicago, III: 1960). 1979;97(11):2192-6.

2. Lopez-Garcia JS, Garcia-Lozano I, Gimenez-Vallejo C, Jimenez B, Sanchez A, de Juan IE. Modified lateral tarsal strip for involutional entropion and ectropion surgery. Graefes Arch Clin Exp Ophthalmol. 2017;255(3):619-25.

3. Lee H, Hwang JY, Kim JW, Park M, Baek S. The effectiveness of simultaneous lateral tarsal strip with endonasal dacryocystorhinostomy for the treatment of nasolacrimal duct obstruction and lower lid laxity. J Craniofac Surg. 2013;24(3):980-3.

4. Lee H, Lee JS, Chang M, Park M, Baek S. The effectiveness of simultaneous medial spindle and/or lateral tarsal strip procedure in East Asian patients who need endonasal dacryocystorhinostomy. Graefes Arch Clin Exp Ophthalmol. 2013;251(12):2789-96.

5. Kam KY, Cole CJ, Bunce C, Watson MP, Kamal D, Olver JM. The lateral tarsal strip in ectropion surgery: is it effective when performed in isolation? Eye (Lond). 2012;26(6):827-32.

6. Anderson RL. Tarsal strip procedure for correction of eyelid laxity and canthal malposition in the anophthalmic socket. Ophthalmology. 1981;88(9):895-903.

7. Chang L, Olver J. A useful augmented lateral tarsal strip tarsorrhaphy for paralytic ectropion. Ophthalmology. 2006;113(1):84-91. 
8. Ryu WY, Suh JY, Ahn HB. Lateral Tarsal Strip Procedure and Silicone Tube Intubation on the Functional Nasolacrimal Duct Obstruction. J Korean Ophthalmol Soc. 2010;51(9):1174-8.

9. Rabinovich A, Allard FD, Freitag SK. Lower eyelid involutional entropion repair with lateral tarsal strip and infraciliary rotation sutures: surgical technique and outcomes. Orbit. 2014;33(3):184-8.

10. Pascali M, Avantaggiato A, Carinci F. Tarsal Strip Versus Tarsal Belt in Ectropion Correction: A Statistical Evaluation. J Craniofac Surg. 2017;28(2):e186-e9.

11. Barrett RV, Meyer DR. The modified Bick quick strip procedure for surgical treatment of eyelid malposition. Ophthalmic Plast Reconstr Surg. 2012;28(4):294-9.

12. Vydláková J, Tesař J, Krátký V, Šín M, Němec P. LATERAL TARSAL STRIP TECHNIQUE IN CORRECTION OF EYELID ECTROPION AND ENTROPION. Ceska a slovenska oftalmologie: casopis Ceske oftalmologicke spolecnosti a Slovenske oftalmologicke spolecnosti. 2021;77(2):73-8.

13. Goddard L, Chesnut C. Simultaneous Lateral Tarsal Strip and Medial Spindle Procedures for Cicatricial Ectropion. Dermatologic surgery: official publication for American Society for Dermatologic Surgery [et al]. 2019;45(7):898-905.

14. Ko SJ, Kim SD. Involutional Ectropion Repair with the Modified Medial Spindle and the Lateral Tarsal Strip Procedure. J Korean Ophthalmol Soc. 2012;53(2):187-92.

15. Rosenstock T, Hurwitz JJ. Functional obstruction of the lacrimal drainage passages. Canadian journal of ophthalmology Journal canadien d'ophtalmologie. 1982;17(6):249-55.

16. Vick VL, Holds JB, Hartstein ME, Massry GG. Tarsal strip procedure for the correction of tearing. Ophthalmic Plast Reconstr Surg. 2004;20(1):37-9.

17. Della Rocca DA. The lateral tarsal strip: illustrated pearls. Facial Plast Surg. 2007;23(3):200-2.

\section{Figures}



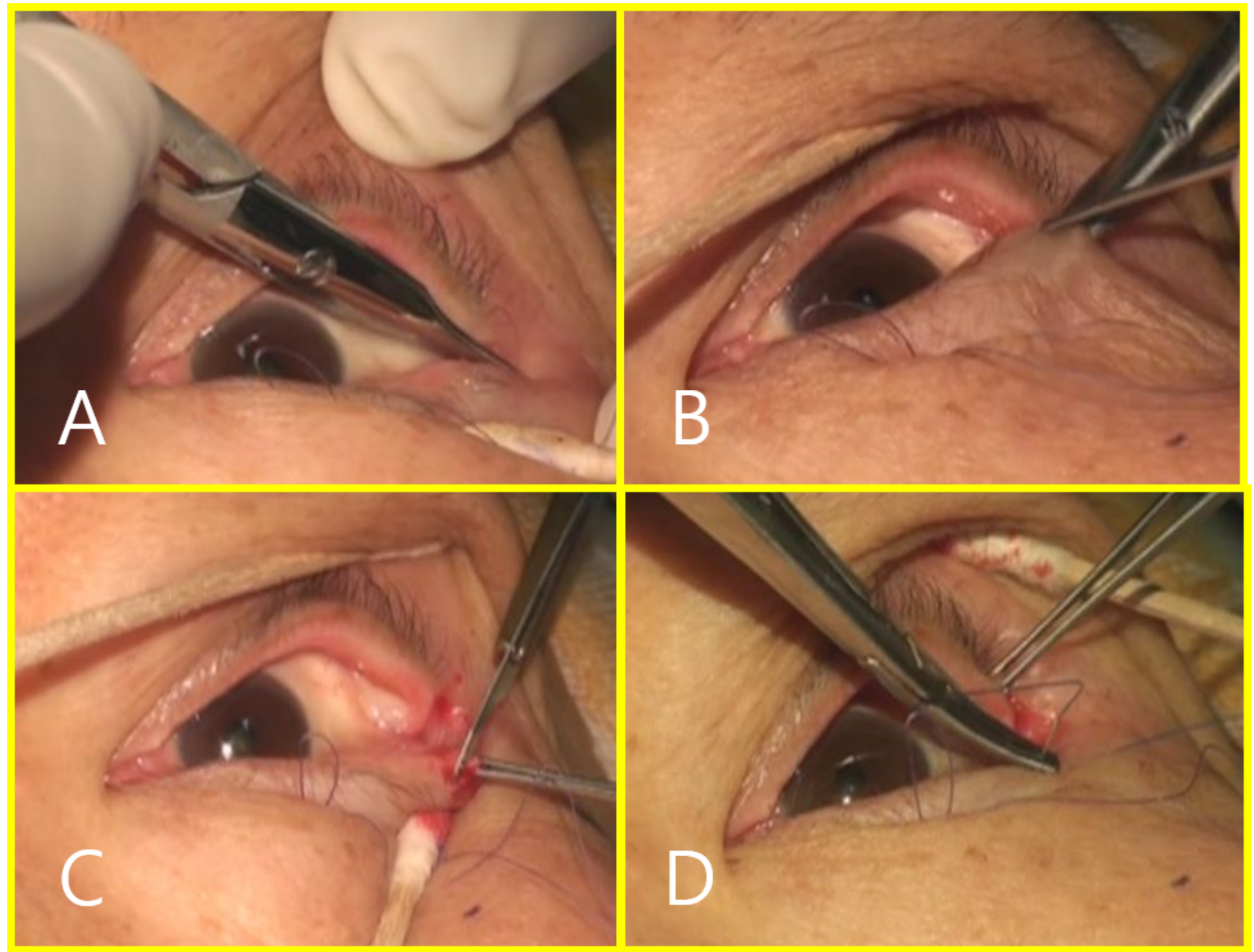

\section{Figure 1}

The lateral tarsal strip procedure

(A) Lateral canthotomy and inferior cantholysis were performed.

(B) Grasp the lateral aspect of the lower eyelid with Adson' forceps and make a deep incision through the orbicularis muscle to the orbital rim using Steven's scissors.

(C) A "lateral tarsal strip" is made by stripping the mucocutaneous junction tissue, removing the conjunctival epithelium, and excising the anterior lamella using a blade No.15.

(D) A "lateral tarsal strip" is attached to the internal lateral orbital rim with a double-armed 4-0 polyglactin suture. A deeply buried 7-0 polyglactin suture is used to close the lateral canthal orbicularis muscle. Skin closure is performed with interrupted 7-0 black silk sutures. 

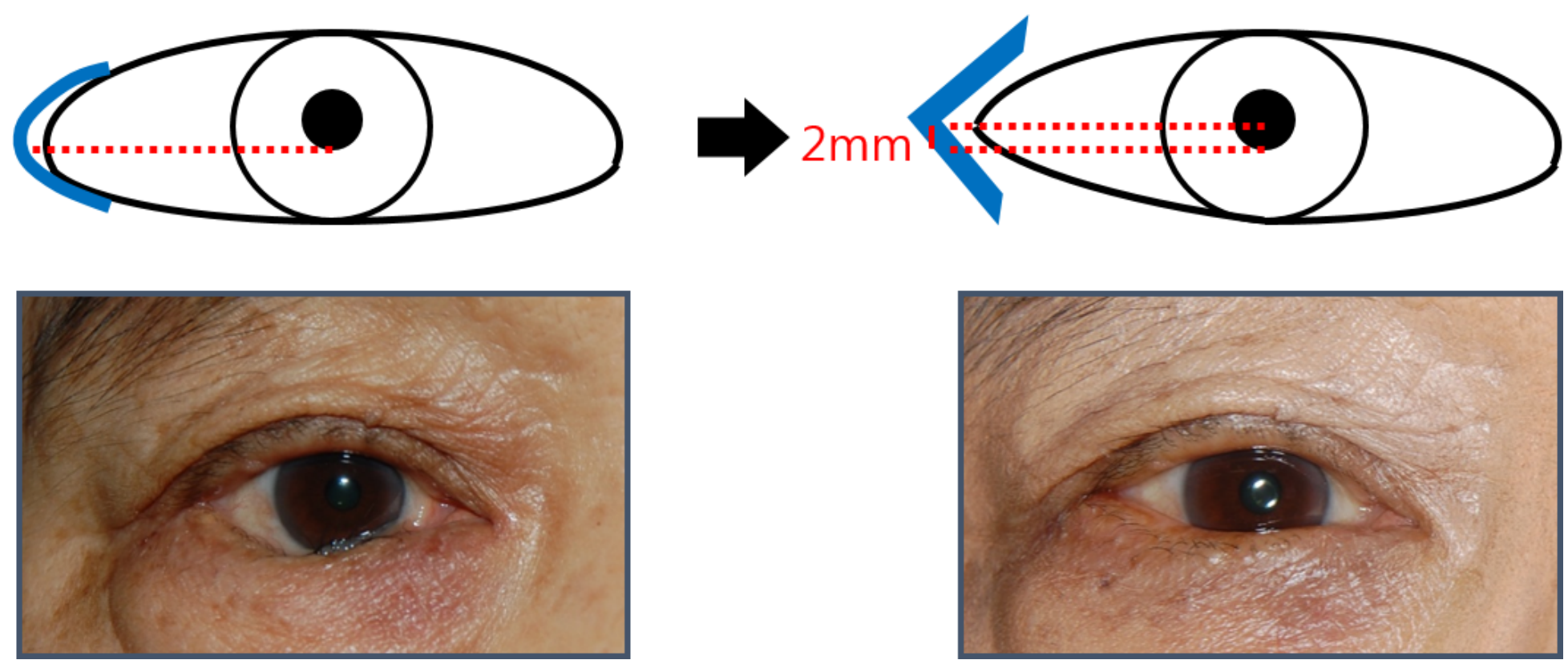

Figure 2

Schematic figures and patient imaging

We attached the lateral tarsal strip $2 \mathrm{~mm}$ above the lateral canthal angle. The amount of lateral tarsal strip tightening required depends on the fixation point height at the internal orbital rim. The patient showed preoperative involutional entropion on the right lower eyelid. Six months after surgery, the patient showed no recurrence.

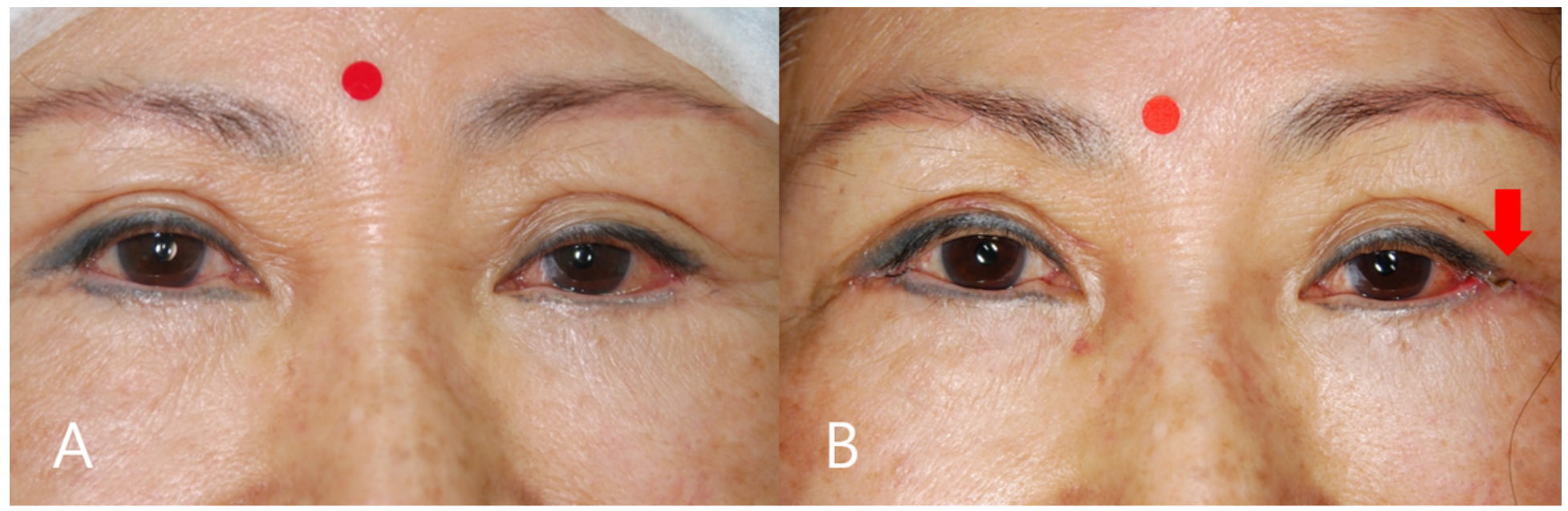

Figure 3

Patient imaging

(A) The patient showed preoperative involutional ectropion on the left lower eyelid.

(B) Three months after surgery, the patient revisited our clinic because of recurrence (arrow).

Undercorrection with the lower height of the lateral tarsal strip fixation was the main cause of recurrence. 


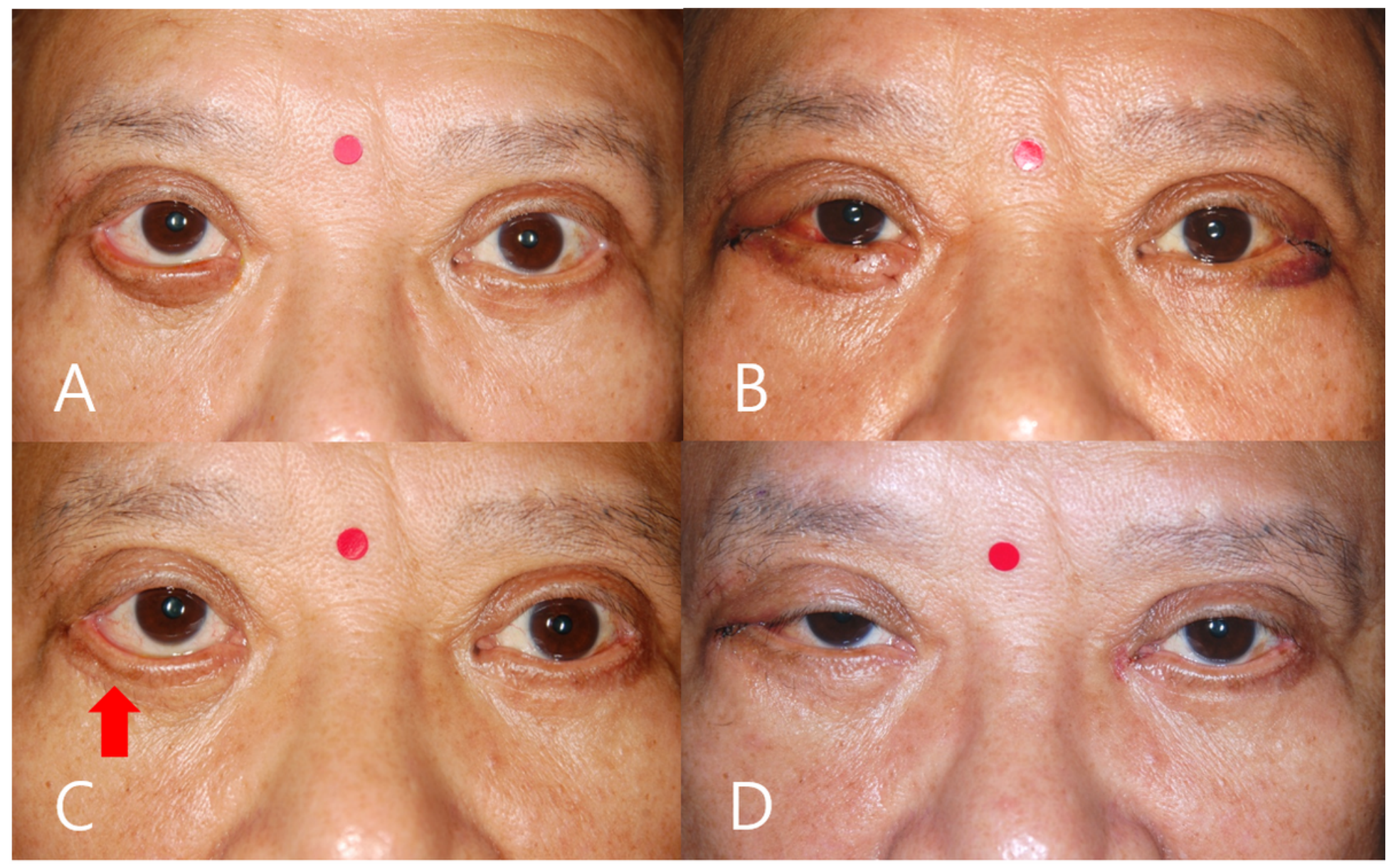

\section{Figure 4}

\section{Patient imaging}

(A) The patient showed preoperative lower lid retraction on both lower eyelids after undergoing lower blepharoplasty at another clinic.

(B) One week after surgery, the patient showed no acute complications.

(C) However, one month after surgery, the patient showed recurrence on the right lower eyelid (arrow) and planned to undergo lateral tarsal strip revision with overcorrection.

(D) Six months after revision surgery, the patient showed no recurrence. 


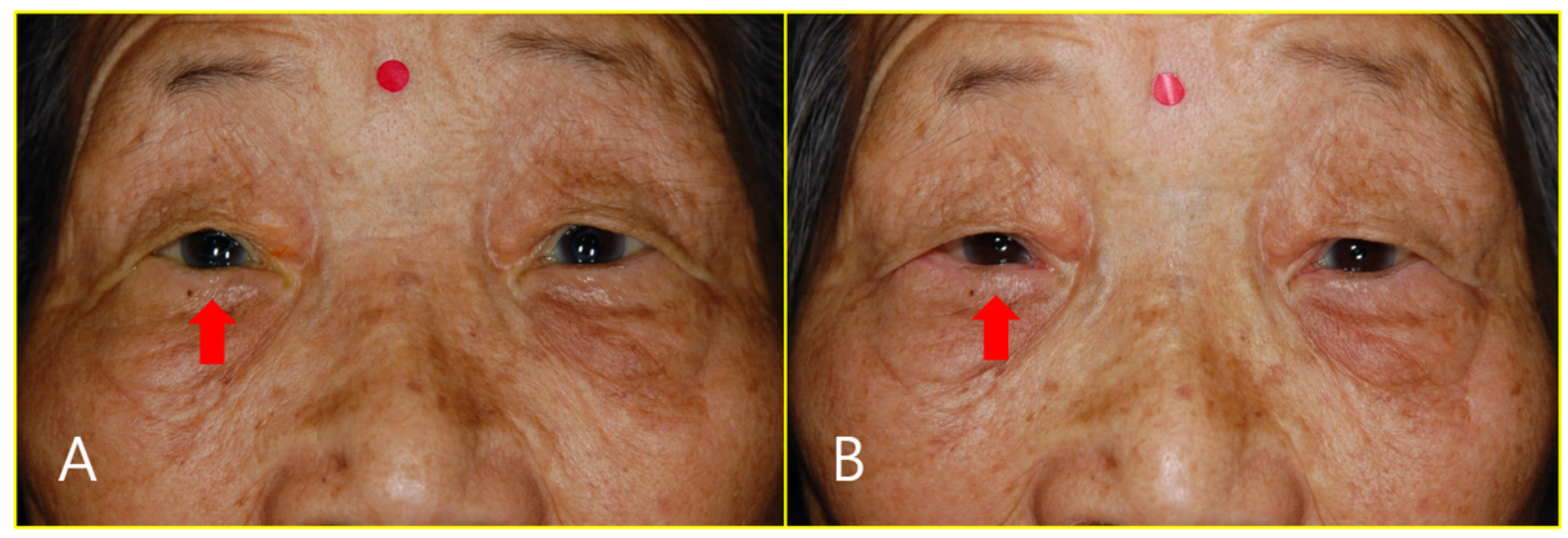

\section{Figure 5}

\section{Patient imaging}

(A) The patient showed involutional entropion with orbicularis overriding on the right lower eyelid (arrow). We performed lateral tarsal strip with skin muscle excision.

(B) Six months after surgery, the patient showed no recurrence.
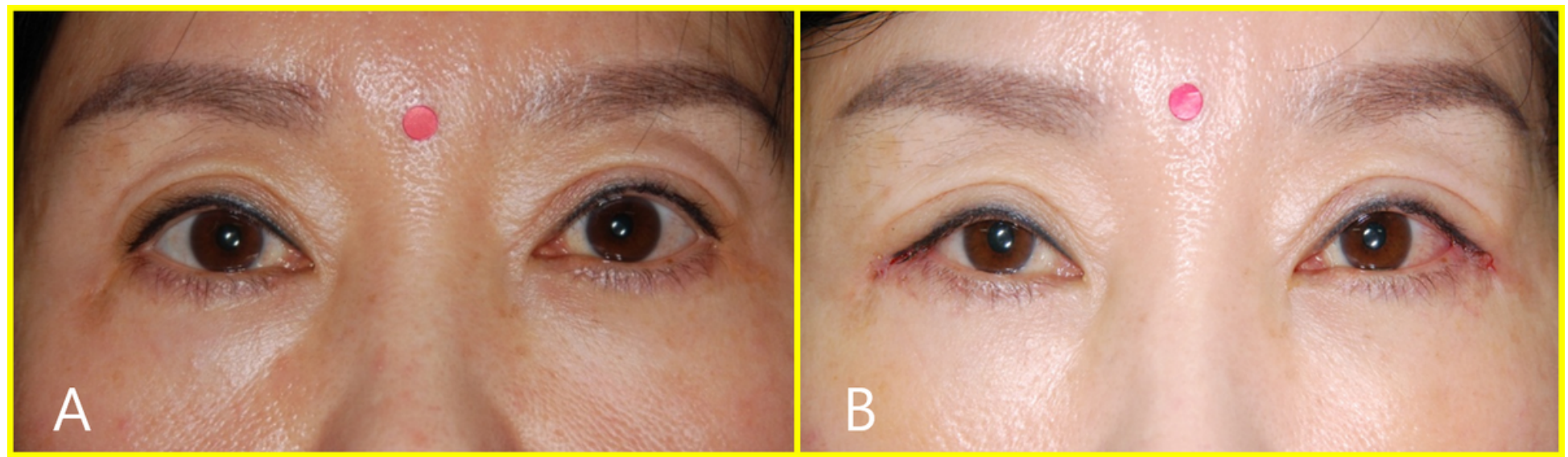

\section{Figure 6}

Patient imaging

(A) The patient showed lower lid laxity on both lower eyelids and functional epiphora.

(B) Six months after surgery, the patient showed no recurrence or epiphora. 


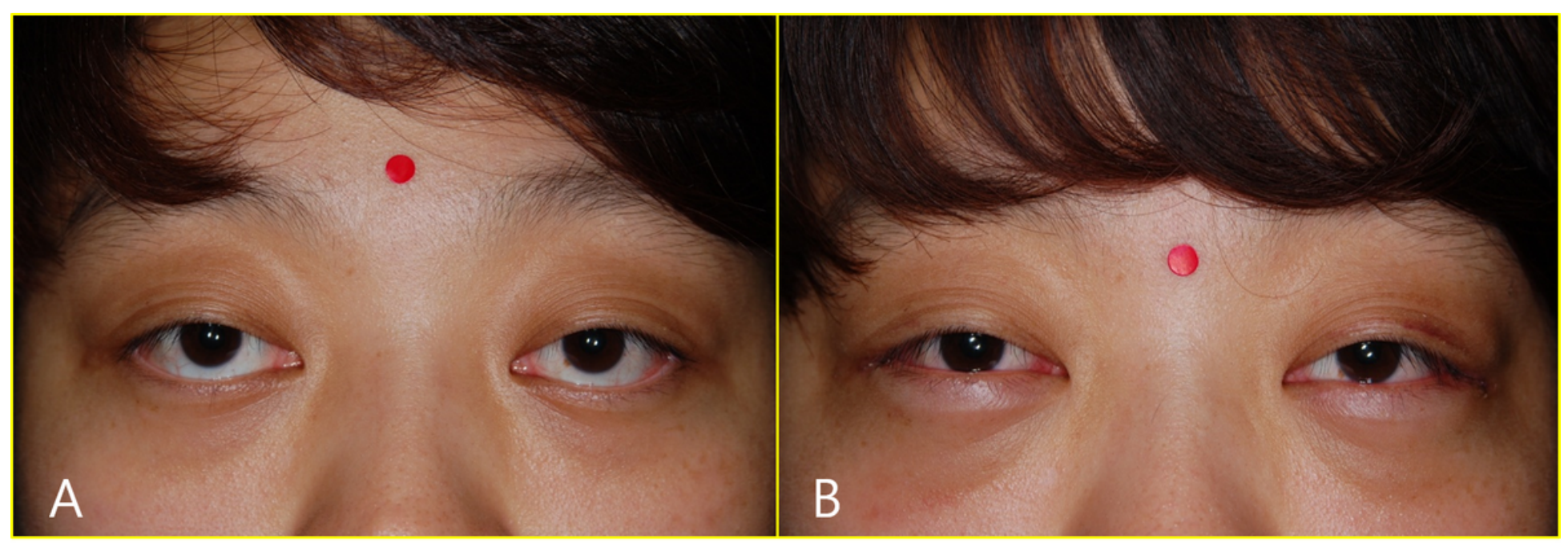

\section{Figure 7}

\section{Patient imaging}

(A) The patient showed lower lid retraction on both lower eyelids and functional epiphora.

(B) Six months after surgery, the patient showed no recurrence or epiphora.

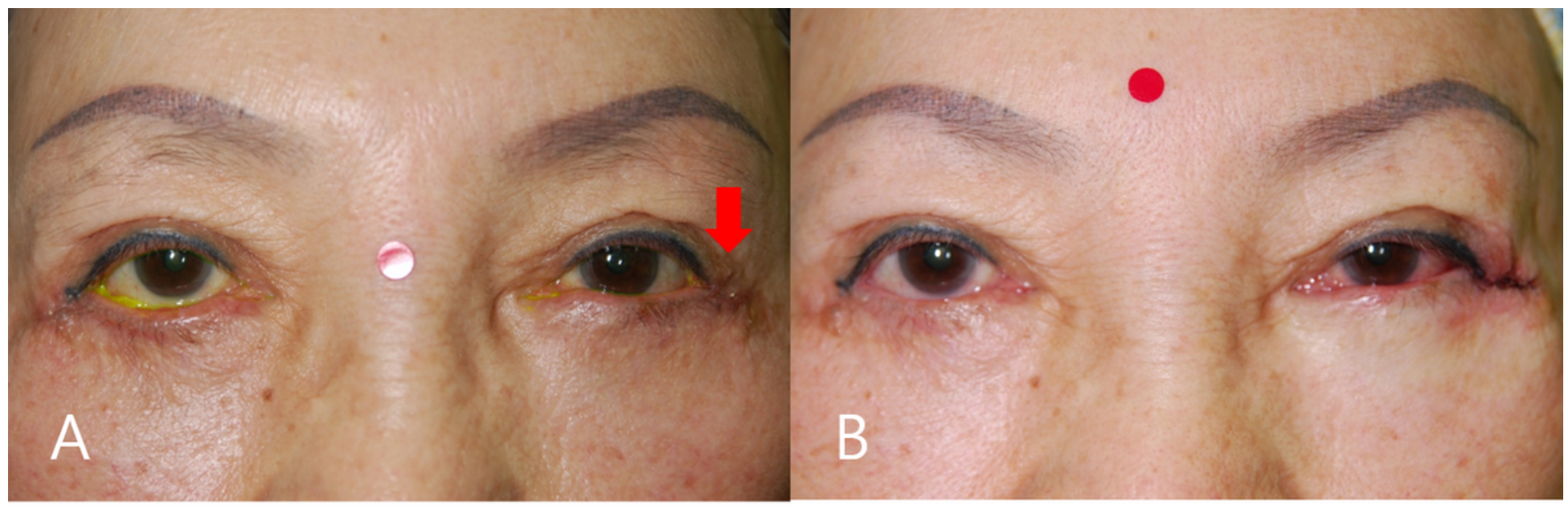

\section{Figure 8}

Patient imaging

(A) One month after undergoing lateral tarsal strip, lateral webbing was found on the left canthus. We performed revision lateral tarsal strip to correct the webbing.

(B) One month after revision surgery, the patient showed no complications. 


\section{Supplementary Files}

This is a list of supplementary files associated with this preprint. Click to download.

- 4.TablesSupplementaltarsalstripfinal.docx 